\title{
Virtual simulation analysis and verification of seed-filling mechanism for dipper hill-drop precision direct rice seeder
}

\author{
Wang Jinwu ${ }^{1 *}$, Zhou Wenqi ${ }^{1}$, Tian Liquan ${ }^{2}$, Li Shuwei ${ }^{1}$, Zhang Zhao ${ }^{1}$ \\ (1. College of Engineering, Northeast Agricultural University, Harbin 150030, China; \\ 2. College of Mechanical and Electrical Engineering, Jinhua Polytechnic, Jinhua 321017, China)
}

\begin{abstract}
In order to improve the seeder' seed-filling ability of the dipper hill-drop precision direct rice seeder, and to meet the mechanization requirement of high speed operation, the self-designed seeder was taken as the objective to explore its seed-filling mechanism and the movement status of rice seed in seed box from the perspective of mechanics. The force models of seed-filling process by dipper were established, and the influential regularity of its rotation speed to compressive resistance of seed population was analyzed as well. The image processing Module-Clipping of discrete element simulation software EDEM was used in the virtual simulation analysis for the process of the seed filling into the dipper, and the velocity relation curve and the force changing curve between rotation speed and seeds were obtained. According to the virtual experiment, the composite filling force of seeds, i.e. the qualified rate on filled rice seed amounts was the largest when rotation speed was at $40 \mathrm{r} / \mathrm{min}$. The performance test bed of seeder was used to verify the simulation results, in which the qualified rate on scooped rice seed amounts was taken as the index, and six rotation speeds of seed-filling dipper were also selected for analysis of seed-filling ability of the device. The results are as follows: with the increase of working speed, the qualified rate on filled rice seed amounts fluctuated with a trend of cosine curve, the largest value was $94.16 \%$ occurred when the rotation speed of seed-filling dipper was at $40 \mathrm{r} / \mathrm{min}$. The variation trend of simulation value was approximately consistent with that of verification value. The study can provide a reference for the research and development of mechanical seeder.
\end{abstract}

Keywords: precision direct rice seeding, rice seed, dipper seeder, seed-filling performance, EDEM, force model DOI: $10.25165 /$ j.ijabe.20171006.2981

Citation: Wang J W, Zhou W Q, Tian L Q, Li S W, Zhang Z. Virtual simulation analysis and verification of seed-filling mechanism for dipper hill-drop precision direct rice seeder. Int J Agric \& Biol Eng, 2017; 10(6): 77-85.

\section{Introduction}

Direct seeding in hill of rice is a cultivation pattern that allows seeds to be directly sown into the paddy field, which can conserve water resources, save seeding areas

\section{Received date: 2016-10-12 Accepted date: 2017-04-18}

Biographies: Zhou Wenqi, PhD candidate, research interests: design of agricultural mechanization equipment, Email: zhouwenqi1989@163.com; Tian Liquan, PhD, Lecturer, research interests: farm machine and rice production mechanization, Email: tiqbuct@sohu.com; Li Shuwei, Master candidate, research interests: agricultural mechanization engineering, Email: 274783068@ qq.com; Zhang Zhao, Master candidate, research interests: agricultural mechanization engineering, Email: 1150154508@qq.com. *Corresponding author: Wang Jinwu, PhD, Professor, research interests: farm machine and mechanical reliability. College of Engineering, Northeast Agricultural University, Harbin 150030, China. Tel: +86-451-55191188, Email: jinwuw@163.com. and lower labor intensity. It has been proved a significant method for planting efficient and high-quality rice $^{[1-3]}$.

The precision direct rice seeder for hill-drop is a crucial aspect in direct seed-metering machines of rice, and seed-metering performance is a critical factor in rice-sowing technology. Besides, the qualified rate of seed-filling has an important influence on the seed-metering performance ${ }^{[4,5]}$.

In foreign countries, mechanical seeders play a vital role. For instance, Maleki et al. ${ }^{[6,7]}$ designed aspiral-grooved direct seed-metering device. Kumara et al. ${ }^{[8]}$ designed a horizontally rotated cylindrical drum of drum seeder. With wheels rotating, drums are rotating and seeds are filled inside the drum from its orifices. It relied on passive seed-filling to complete the seed-filling 
process. This method will lead to the reduction of qualified rate of seed-filling, and the study does not analyze the influential regularity of composite filling force on qualified rate of seed-filling, as it can enhance the performance of seed-filling. In China, Yi et al. ${ }^{[9]}$ designed a bowl-tray rice precision seeder and optimized the mechanism parameters to improve its performance. The seeder is based on rice seeds that fall upon the surface of shaped-hole plate due to gravity and down with inertial force; it also relies on seeds being filled passively to complete the seed-filling process. Zhang et al. ${ }^{[10]}$ designed a kind of pneumatic rice seeder. It aspirates seeds to the surface of seed pan forwardly, and finishes the seed-filling process under the self-gravity and air pressure. This device owns advantages such as seed-saving, low rate of seed being-damaged, high qualified rate of seed-filling. However, it cannot meet the higher requirement of cost saving and working accuracy. Moreover, this study does not analyze the influence of composite force including gravity, aspirated-air force and centrifugal force during seed-filling process, which can influence the seed-filling ability. Zhai ${ }^{[11]}$ has studied the relation among average velocity, stress, displacement with the change of time by Pro/E and EDEM, while seed-filling process and mechanism analysis were not involved.

The dipper hill-drop precision direct rice seeder ${ }^{[12]}$ was considered as the research object in this research, the passive way of seed-filling as well as the seed-filling mechanism was elaborated. Furthermore, in order to explore the influences of different working rotation speeds of the dippers on the qualified rate of the seed-filling process, a simulation analysis was conducted to investigate the seed-filling process of the dippers with EDEM, besides, the curve graph between movement velocity and force of seeds in the seed-filling process was demonstrated, and the simulation result was verified with the test bed. The research acquisition is expected to provide a reference for the study on enhancing the working performance of the seeder.

\section{Structure and working principle of seeder}

The structure of the seeder is shown in Figure 1. It mainly consists of guiding roller (1), seed box (2), dippers (3), barrier bar (4), torsion spring (5), rotating base (6), seed-throwing tube (7), seeding shaft (8), etc.

In the operation process, the force is delivered to seeding shaft from chain transmission. The rotating base is driven by the shaft, and rotation direction is shown in Figure 1. It drives dippers evenly distribute on it to dig into the seed population, which ensures seeds to be filled into the dipper quantitatively. At the meantime with the continuous rotation of base, it will be separated from the seed population, thus the seed-filling process is accomplished. Then, it enters the seed-cleaning zone. The unstable seeds in dippers fall into the seed population on account of their own gravity; then the seed-cleaning process is accomplished. Afterwards, the dippers rotate into the guiding zone, and they contact with the guiding roller reciprocally. The roller presses the dipper, making one side of the torsion spring rotate and store angular energy, therefore, the rotary force has been stored And then the dipper clings on the guiding roller to guide and protect seeds. In seed-throwing process, the dipper continues to rotate driven by rotary base, when there is no contact between dippers and guiding roller, torsion spring is supposed to be reset to release angular energy, which can produce rotary force to make dipper rotate clockwise and collide with barrier bar. Thus, seeds in the dippers will be rebounded through throwing tube on the paddy field, and then the seed-throwing process is fulfilled.

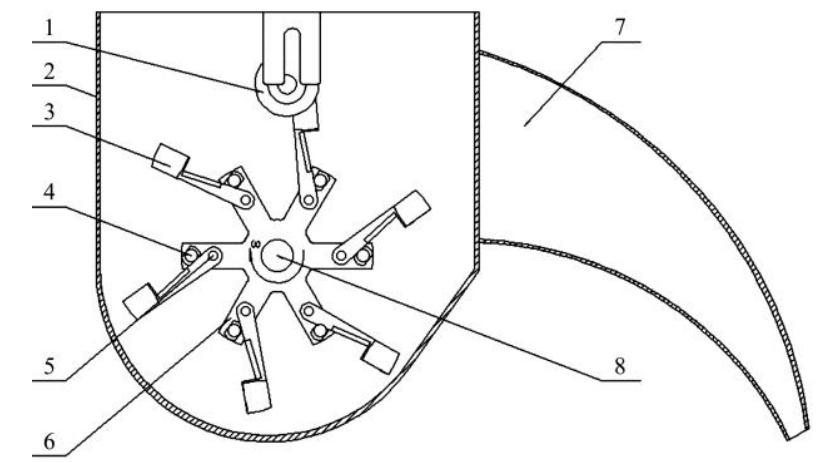

1. Guiding roller 2. Seed box 3. Dipper 4. Barrier bar 5. Torsion spring 6. Rotating base 7. Seed-throwing tube 8 . Seeding shaft

Figure 1 Structure of dipper hill-drop precision direct rice seeder

\section{Force analysis of seed-filling process}

The process of seed-filling is one of the most critical aspects in the entire seed-metering process. The seed-filling precision of dipper can improve the 
seed-metering performance. Thus, the force analysis of seed-filling process is in dispensable. Figure 2 shows the force status in the seed-filling process.

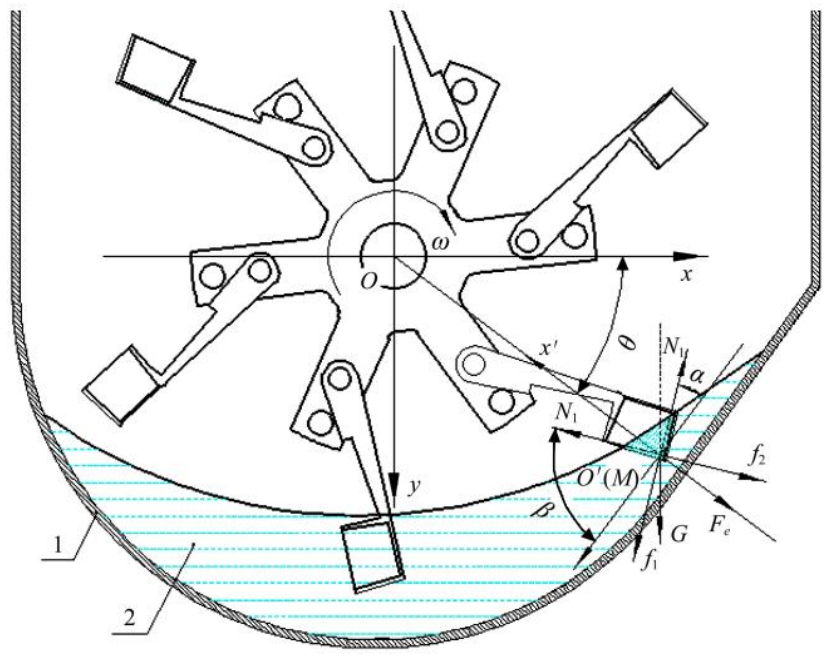

1. Wall of seed box 2. Seed population

Figure 2 Force analysis in process of seed-filling

When the dipper digs into seed population, it drives seed population to move. Therefore, an interface must be produced between the dynamic seeds and the static seeds in seed population, which is called external slip surface of seeds outside the dippers; when seeds are filled into the dippers, there is an interface between seeds and inner layer of the dipper or seeds in the dipper, which is called inner slip surface of seeds. The place where seeds exist between two surfaces is called sliding bodies. To simplify analysis process, the two sliding surfaces and free surface of sliding bodies are all set to plane. The angle between external slip surface and tangent of $\mathrm{M}\left(O^{\prime}\right)$ point's motion trajectory on the dipper is slip angle $\alpha$, and the angle between the inner slip surface and tangent of $M$ point's motion trajectory on the dipper is slip angle $\beta$. In the seed-filling process of dippers, sliding bodies slide along internal surface of dippers until they get to the bottom of them due to the effect of filling force. The seeds are restricted in this position, the slip angle $\alpha$ increases, while the slip angle $\beta$ gradually decreases with the increase of filling numbers of seeds. When the filling rate is higher, the slip angle $\beta$ can be reduced down to 0 at last. At this time, the seeds stop filling into the dipper. The sliding body is in a relatively static state when the seeds are contacted with the bottom of the dippers. xoy as static coordinate system was established, the rotation center was defined in coordinate origin as $o$.
The acting force of seeds at M point consists of gravity $G$, support force $N_{1}$ on the internal slip surface, pressure $N_{2}$ on external slip surface, inertia centrifugal force $F_{e}$ and friction forces $f_{1}$ and $f_{2}$ on both slip surfaces. The sliding bodies are in a state of balanced force and at a relatively static stage. The dynamic coordinate system $x^{\prime} o^{\prime} y^{\prime}$ was established, tangential direction of M point's motion trajectory on the dipper was defined as $y^{\prime}$ axis forward direction, normal direction of the motion trajectory which points to coordinate origin $o^{\prime}$ as $x^{\prime}$ axis forward direction. Thus, the balance equations were shown, as follows:

$$
\left\{\begin{array}{l}
N_{1} \cos \alpha+N_{2} \cos \beta-F_{e}-f_{1} \sin \alpha-f_{2} \sin \beta-G \sin \theta=0 \\
N_{1} \sin \alpha+f_{1} \cos \alpha+G \cos \theta-f_{2} \cos \beta-N_{2} \sin \beta=0 \\
F_{e}=m r \omega^{2} \\
G=m g \\
f_{1}=\mu_{1} N_{1} \\
f_{2}=\mu_{2} N_{2}
\end{array}\right.
$$

where, $m$ is the mass of seed, $\mathrm{kg} ; r$ is the rotation radius, $\mathrm{m} ; \alpha, \beta$ are the slip angle, $\left(^{\circ}\right) ; \mu_{1}$ is the friction coefficient between seeds and dipper layer; $\mu_{2}$ is the internal friction coefficient among seeds; $\omega$ is the angular speed of dipper, $\mathrm{rad} / \mathrm{s} ; \theta$ is the initial position angle, $\left({ }^{\circ}\right)$.

In order to simplify the calculation process, assumed that $\mu_{1}=\mu_{2}=\mu$, therefore Equations (1) would be changed to the Equation (2):

$$
\left\{\begin{array}{l}
N_{1}=\frac{m\left(r \omega^{2}+g \sin \theta\right)[(\mu \cos \beta+\sin \beta)-m g \cos \theta]}{\left(1-\mu^{2}\right) \sin (\alpha+\beta)+2 \mu \cos (\alpha+\beta)} \\
N_{2}=\frac{m\left(r \omega^{2}+g \sin \theta\right)(\sin \alpha+\mu \cos \alpha)}{\left(1-\mu^{2}\right) \sin (\alpha+\beta)+2 \mu \cos (\alpha+\beta)}
\end{array}\right.
$$

The sliding bodies are filled into the dipper, the filling force $F_{c}$ is:

$$
F_{c}=N_{2}-\mu N_{1}-G \sin (\beta+\theta)-F_{e} \cos \alpha
$$

where, $F_{c}$ represents the filling force of seeds, $\mathrm{N}$.

Through the force analysis of seed-filling process and Equation (3), we can have a better understanding that when structure parameters of the seeder and parameters of the seed are constant, the filling force is associated with angular speed and initial position angle of the dippers. In the field experiment, the height of seed population was controlled by the distance between seed-entering tube and bottom of seed box, thereby 
making initial position angle between the dippers and seed population was a fixed value. Accordingly, only the rotation speed of the dippers was taken into account and other working parameters were fixed. With this prerequisite, the discrete element software EDEM was utilized to make virtual simulation on process of seed-filling and the test bed was also carried out to verify the simulation.

\section{EDEM discrete element simulation analysis}

\subsection{Simulation model and parameters setting}

In order to verify the rationality of the force model on seed-filling process, the simulation model and parameters should be set in the preprocessed module of EDEM before the virtual simulation test of seed-filling process.

1) Establishment of seed model

The shape and size of seed are significant to its flowing and filling status in the seeder. In order to simulate the state of seeds, the method of combination of multiple spheres was adopted in filling process, which was used to more accurately simulate the working process of device and the movement state of seeds. In order to simplify the model, various radius of spherical particles were selected to represent the model of shape-approximate seeds $^{[13]}$. For No.3 Longqing direct-seeding rice, 100 seeds were randomly selected and their sizes were measured as well. Then, the mean value of seeds dimension can be obtained (7.75 $\mathrm{mm} \times$ $3.11 \mathrm{~mm} \times 2.12 \mathrm{~mm})$. According to the size data, the simulation model of the seed was established by the particles module $^{[14-17]}$ of EDEM, as shown in Figure 3.



Figure 3 Model of rice seed

In order to simplify the parameters of virtual model, Poisson's ratio, elastic modulus, density as well as other physical parameters were considered as fixed value in simulation process.

\section{2) Establishment of seeder}

Firstly, certain components irrelevant to the contact between the seeder and seeds during working process were excluded. Then a three-dimensional software CATIA was used to construct the model of seeder, the model was imported into Geometry option of EDEM in .stp format, and the discrete element model was established under the condition that the parameters and material characteristic were well set. Besides, the seed box and rotating base were set in mesh form so that the movement of seed during seed-filling can be clearly observed; the results are shown in the following Figure 4.

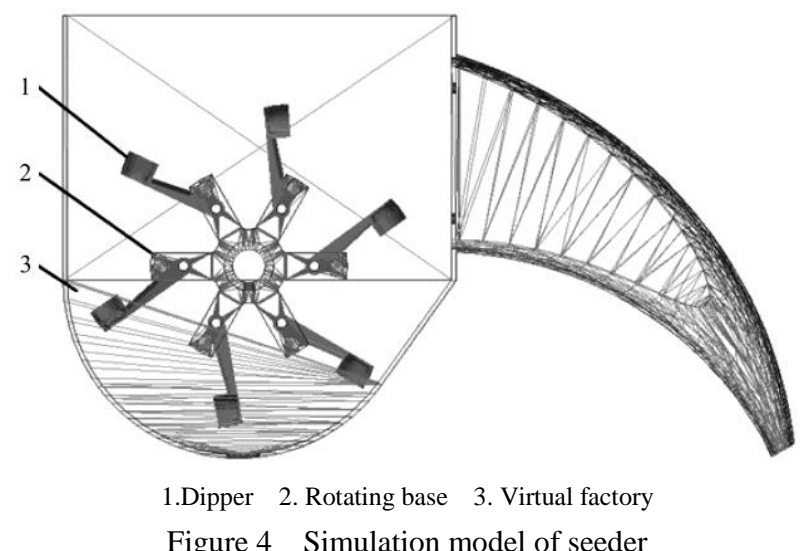

3) Setting of global variables

As the surface of seeds is non-adhesive after awn-cleaning, seed soaking and germination-accelerating, the contacts between the seeds, the seed and the shell of seeder were set as non-slip contact model. Parameters were shown in Table $1^{[18,19]}$.

Table 1 Setting of the global variables

\begin{tabular}{clc}
\hline \multicolumn{1}{c}{ Item } & \multicolumn{1}{c}{ Parameter } & Value \\
\hline \multirow{2}{*}{ Seed } & Poisson's ratio & 0.25 \\
& Elastic modulus/Pa & $3.75 \times 10^{8}$ \\
& Density $/ \mathrm{kg} \cdot \mathrm{m}^{-3}$ & 1150 \\
\hline \multirow{2}{*}{ Stainless steel shell } & Poisson's ratio & 0.29 \\
& Elastic modulus/Pa & $7.5 \times 10^{10}$ \\
& Density/kg $\cdot \mathrm{m}^{-3}$ & 8000 \\
\hline \multirow{2}{*}{ Seed-seed } & Restitution coefficient & 0.6 \\
& Static friction coefficient & 0.3 \\
& Dynamic friction coefficient & 0.02 \\
\hline \multirow{2}{*}{ Seed-shell } & Restitution coefficient & 0.5 \\
& Static friction coefficient & 0.52 \\
& Coefficient of Rolling Friction & 0.01 \\
\hline
\end{tabular}

4) Setting of other parameters

In the simulation process, $15 \%$ of the time step of Rayleigh was regarded as the fixed time step. The time 
of simulation was the same as that of rotating process.

The time interval of data writing was $0.01 \mathrm{~s}$, and the mesh size was 2 times larger than the smallest seed ${ }^{[20]}$.

Using the latest image processing technology (Clipping) $^{[21]}$ of analyst option in the EDEM, we amputated and removed half of the entire structure along midline of the seed box and dippers by plane cutting, but the contact and bond between seeds, seeds and dipper, the seeds and box of the parts should not be cut. Figures $5 \mathrm{a}-5 \mathrm{j}$ demonstrate the simulation in each phase of seed-filling process, and the distribution of movement shape of seeds in the process. When the dipper did not rotate, all the seed models would remain static condition. With the dipper digging into seed population, seed models slid along the wall of dipper until they get to the bottom. At this time, they were confined in where they stay, and seeds continued to fill into the dipper along the inner slip surface of seeds. The movement shape of seed-filling in EDEM simulation process was consistent with that in analysis of force models.

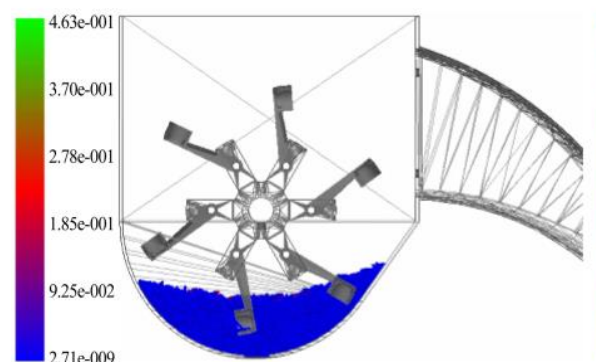

a

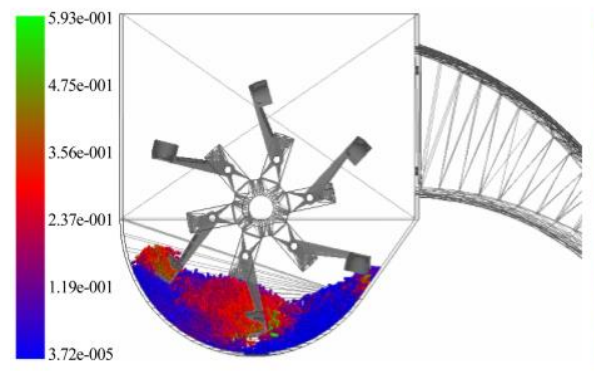

d

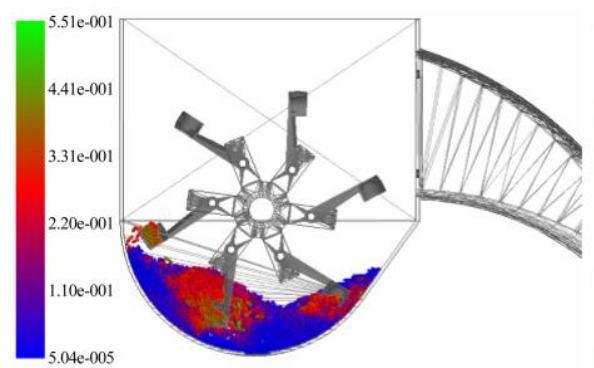

g

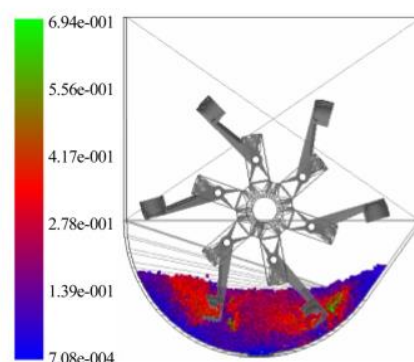

b

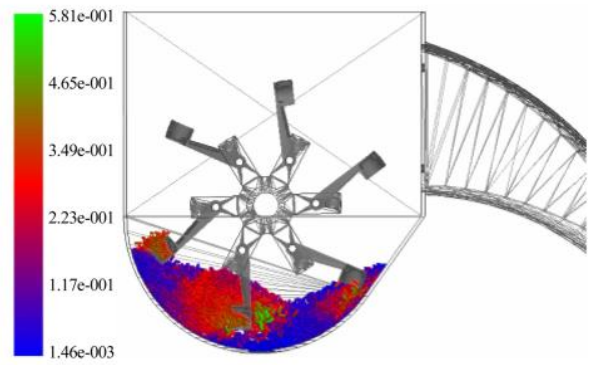

e

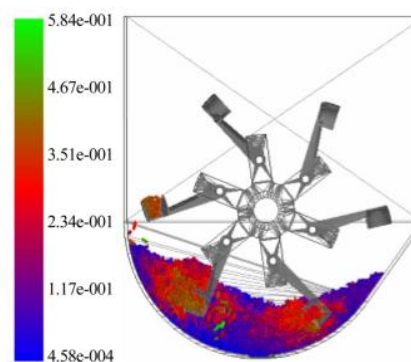

h

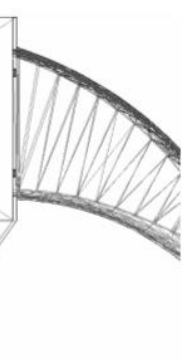

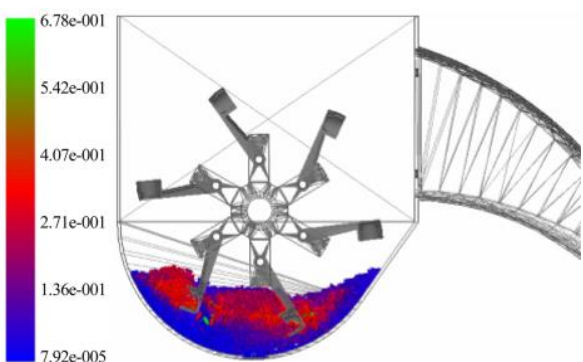

c

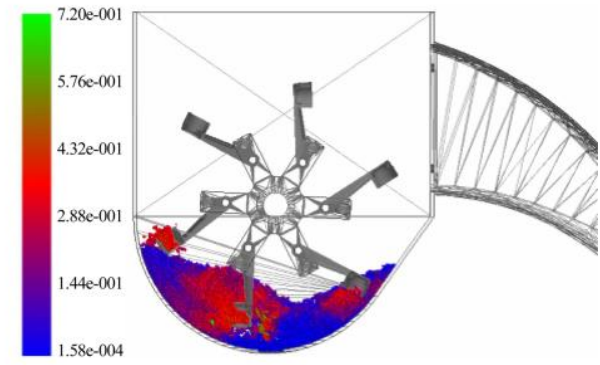

f
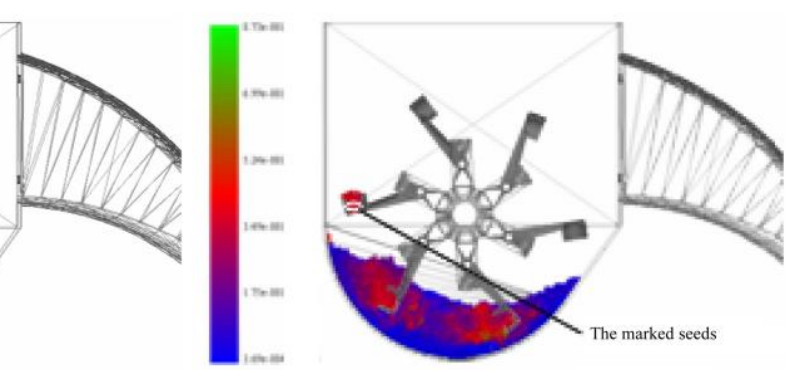

Figure 5 Movement simulation of rice seeds in working process

\subsection{Analysis of simulation results}

In process of simulation and calculation, the movement of each seed model can be displayed in streamline form, and we employed various colors to represent the change of movement speed of seeds in different time. Among them, the blue seed model has the lowest speed, and with the red one followed, while the green seed has the fastest speed. The seeds filled into the dippers were marked, and produced the relation curve between filling speed of seeds and time, which is shown in Figure $5 \mathrm{j}$.

Figure $6 \mathrm{a}$ reveals the relation between the average speed of marked seeds model in same dipper and time when the rotation speed is at $40 \mathrm{r} / \mathrm{min}$. It can be seen that the average speed of each marked seed was consistent with the trend of time. The time before $1.43 \mathrm{~s}$ was a period when virtual factory generated seeds models, and the model of seeds fall into the bottom of the seed box and gradually accumulated after generated. The 
time after $1.43 \mathrm{~s}$ was a period of seed-filling of the dippers. The rotation of dippers drove surrounding seeds to move. A portion of seed model moved towards the inner of dipper under the influence of filling force. The average speed of seed models constantly changed and reached the maximum value when the time was at about $1.48 \mathrm{~s}$, then the speed changed constantly again, and the speed fluctuation condition tended to be gentle after $1.63 \mathrm{~s}$. Figure $6 \mathrm{~b}$ shows the relation between the average speed of marked seed models in same dipper and the time when rotation speed is different. The time of relatively stable state when seeds filled into the dippers was decreasing with the increasing rotation speed, however, the centrifugal force of the seeds was increasing when the rotation speed continued to increase, and the collision between seeds, seeds and layer of the dippers could be intensified, which bounced some seeds and evidently change the speed, moreover the average velocity of seeds fluctuated significantly. Thus, it proves that excessive working speed is not conducive to the relative stability of model.

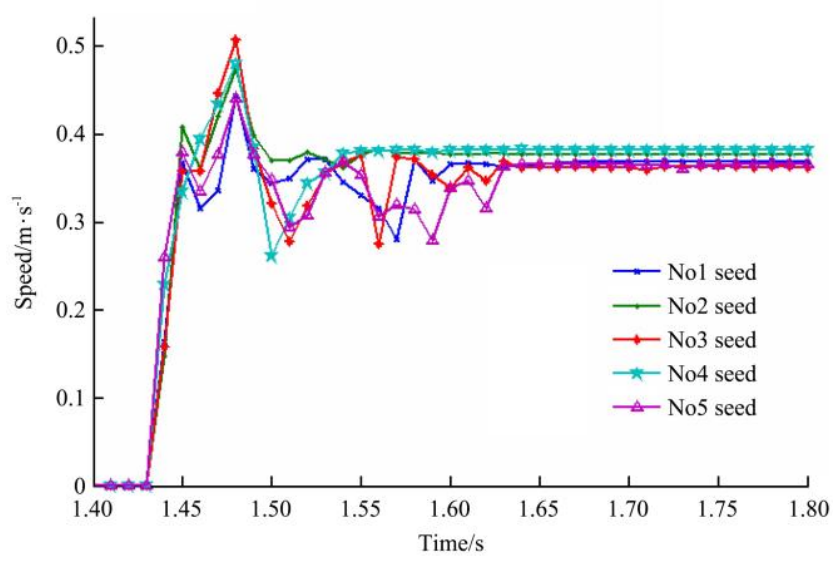

a. Same rotation speed



b. Different rotation speeds

Figure 6 Average speed curve of seeds
It can be seen from the analysis of Equation (3) that seeds were filled into the dippers with the effect of gravity, centrifugal force and the interaction force among seeds. The rotation speed had an effect in filling force of the marked seeds; the relation curve is shown in Figure 7. It can be seen from the figure, the filling force of marked seeds increased at first, then decreased, and its fluctuation trend was inclined to be gentle by degrees with the increase of rotation speed. With a lateral comparison, there was a large gap among maximum value of the seed-filling forces, changing from big to small, when the rotation speed changed from $40 \mathrm{r} / \mathrm{min}$, $50 \mathrm{r} / \mathrm{min}, 30 \mathrm{r} / \mathrm{min}, 60 \mathrm{r} / \mathrm{min}, 70 \mathrm{r} / \mathrm{min}$ to $20 \mathrm{r} / \mathrm{min}$ in turn.

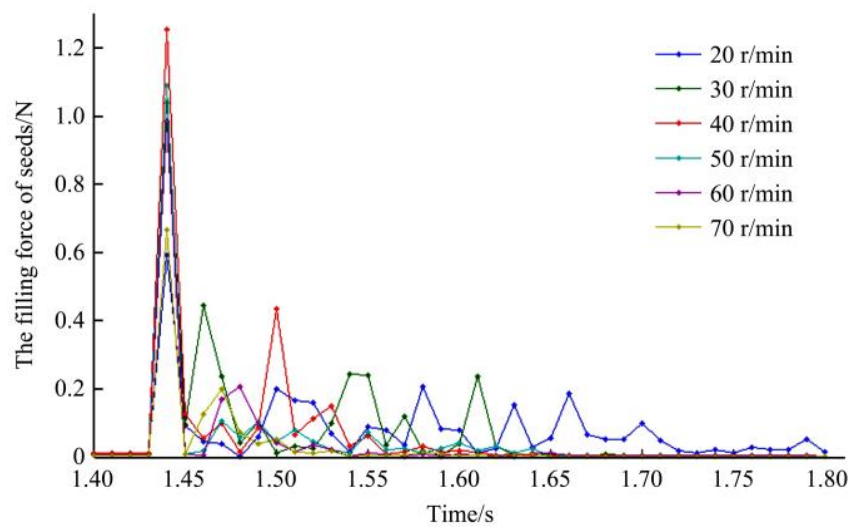

Figure 7 Filling force of seeds at different speeds

When the rotation speed of the dippers was low, the disturbance intensity of dippers to seed population was low. The compressed force of seed population has a remarkable effect on the filling force of sliding bodies of seeds, therefore the larger filling force could be acquired, and it was used for seeds to fill into the dippers. When the rotation speed was high, the disturbance intensity of the dippers to the seed population would increase, and the bigger disturbance intensity could reduce the friction force among seeds. At the same time, the centrifugal force acting upon the sliding bodies would increase. Therefore, the influence of compressed force on sliding bodies of seeds could decrease, and some seeds bounded out of the dippers, which had a certain impact on performance of dippers in quantitative filling.

\section{Experiment and analysis}

\subsection{Experiment}

In order to verify the theoretical analysis and virtual simulation results, and the seed-filling performance of 
seeder was tested, and the No.3 Longqing seed was chosen, the test bed was carried out by JPS-12 computer vision seeder performance test-bed of Northeast Agricultural University. Then the transparent and organic glass plate was selected as the material of seed box and seed-throwing tube for facilitating observation and statistics of the seed-filling process, as shown in Figure 8. And took the rotation speed as the single factor for the experiment design, its values were $20 \mathrm{r} / \mathrm{min}$, $30 \mathrm{r} / \mathrm{min}, 40 \mathrm{r} / \mathrm{min}, 50 \mathrm{r} / \mathrm{min}, 60 \mathrm{r} / \mathrm{min}$ and $70 \mathrm{r} / \mathrm{min}$, and the height of seed-throwing and hill space were $50 \mathrm{~mm}$ and $150 \mathrm{~mm}$ respectively.

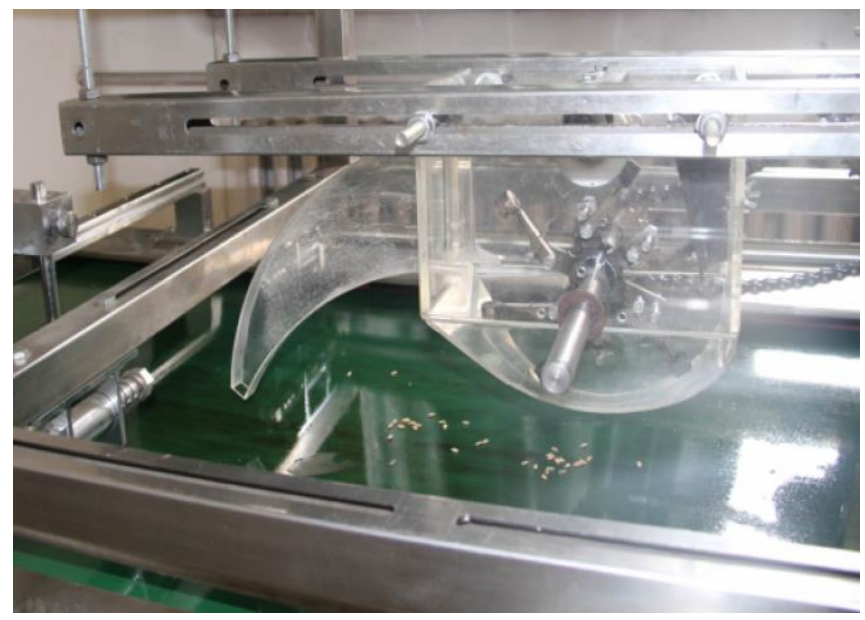

Figure 8 Test bed of seed-filling performance

According to agronomic requirement, the quality standard of conventional rice sowing is 5-8 seeds per hill $^{[22]}$, while it is unqualified when the data is $0-4$ or more than 9 seeds per hill. According to the GB/T6973-2005 test method of the precise sowing ${ }^{[23]}$, the number of seed-throwing was recorded by each dipper continuously. The number of dippers in one group was 250 , and the times that the dipper filled in 5-8 seeds were recorded. The qualified rate of seed-filling process could be calculated by Equation (4):

$$
P_{i}=\frac{\sum_{i=5}^{8} X_{i}}{250} \times 100 \% \quad i=5,6,7,8
$$

where, $P_{i}$ is the qualified rate of seed-filling process, $\%$; $X_{i}$ is the time that a dipper was filled in $i$ seeds.

At the different rotation speeds of the dipper, the simulation values and experiment values about the qualified rate of the seed-filling process are taken as shown in Figure 9.

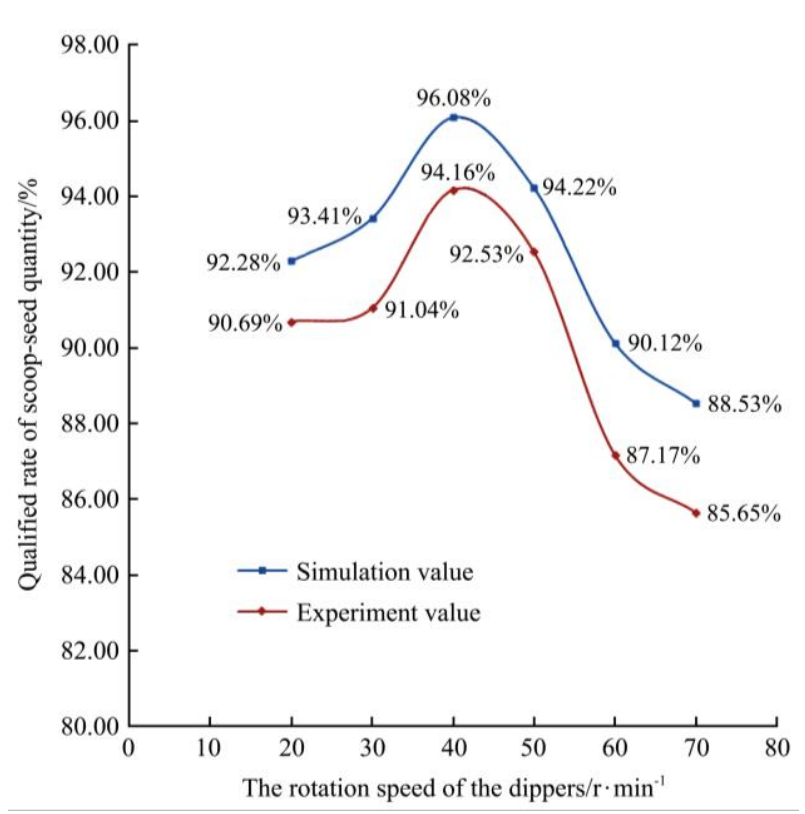

Figure 9 Experiment and simulation results

\subsection{Analysis}

It can be seen in Figure 9 that the simulation values and verification values change regularity of qualified rate of seed-filling are consistent basically. Besides, the minimum relative error between both values is $1.72 \%$, the maximum is $3.27 \%$. When the rotation speed of dipper is at $20-40 \mathrm{r} / \mathrm{min}$, the qualified rate of seed-filling shows increasing trend; and the value achieves maximum when the rotation speed is at $40 \mathrm{r} / \mathrm{min}$, and the verification value and simulation value are $96.08 \%$ and $94.16 \%$, respectively. However, when the rotation speed is at $40-70 \mathrm{r} / \mathrm{min}$, the qualified rate of seed-filling shows a decreasing trend.

It is caused that the seed population compressive resistance has an outstanding effect on filling force when the rotation speed is lower. Furthermore, the dipper stays longer among seed population, which is conductive to seeds filling into the dippers fully. Thus, the qualified rate of seed-filling shows increasing trend; on the contrary, when the rotation speed is larger, centrifugal force of the slide body has a remarkable effect on filling force, which leads the seeds in dippers to be ejected by centrifugal force and then the qualified rate of seed-filling shows decreasing trend.

The reason why simulation values are different from verification values is that the work performance of seeder is not stable, which produces vibration, moreover, the side wall of dippers knocking seeds, and the size of seeds 
exists differences between simulation and reality.

\section{Conclusions}

1) With dipper hill-drop rice precision direct seeder as the research carrier, the force models of seed-filling process were established. From the mechanics view, more filling force can improve the qualified rate of the seeder in the seed-filling process.

2) By using CATIA and EDEM softwares, the three-dimensional models and simulation models of seeder were established to simulate the seed-filling process. The simulation results showed that the seed-filling force changes from big to small when the roation speeds in turn were $40 \mathrm{r} / \mathrm{min}, 50 \mathrm{r} / \mathrm{min}, 30 \mathrm{r} / \mathrm{min}$, $60 \mathrm{r} / \mathrm{min}, 70 \mathrm{r} / \mathrm{min}$ and $20 \mathrm{r} / \mathrm{min}$.

3) By contrasting the results of relationship between the qualified rate and the rotation speed in simulation and experiment, the variation regularity of them was consistent basically. The simulation values and experiment values were maximum when the rotation speed was at $40 \mathrm{r} / \mathrm{min}$, reaching $96.08 \%$ and $94.16 \%$, respectively. Therefore, it was correct and feasible that the qualified rate of seed-filling process was analyzed by using discrete element method.

\section{Acknowledgements}

The authors thank the financial support provided by the National Industry System of Rice Technology of China (CARS-01-44), Heilongjiang Modern Industrial Technology Collaborative Innovation System.

\section{[References]}

[1] Luo X W, Liu T, Jiang E C, Li Q. Design and experiment of hill sowing wheel of precision rice direct seeder. Transactions of the CSAE, 2007; 23(3): 108-112. (in Chinese)

[2] Mahajan G, Chauhan B S, Gill M S. Dry-seeded rice culture in Punjab State of India: Lessons learned from farmers. Field Crops Research, 2013; 144: 89-99.

[3] Peng S B, Yang J C. Current status of the research on high-yielding and high efficiency in resource use and improving grain quality in rice. Chinese Journal of Rice Science, 2003; 17(3): 275-280. (in Chinese)

[4] Rtnayake R M C, Balasoriya B M C P. Re-design, fabrication, and performance evaluation of manual conical drum seeder a case study. Applied Engineering in Agriculture, 2013; 29(2): 139-147.

[5] Yoshinaga S. Improved lodging resistance in rice (Oryza sativa L.) cultivated by submerged direct seeding using a newly developed hill seeder. Jarq-Japan Agricultural Research Quarterly, 2005; 39(3): 147-152.

[6] Maleki M R, Jafari Raufat J V, Mouazen A M, De Baerdemaeker J. Evaluation of seed distribution uniformity of a multi-flight as a grain drill metering device. Biosystems Engineering, 2006; 94(4): 535-543.

[7] Maleki M R, Mouazen A M, de Ketelaerer B, de Baerdemaeker J. A new index for seed distribution uniformity evaluation of grain drills. Biosystems Engineering, 2006; 94(3): 471-475.

[8] Kumar G V P, Srivastava B, Nagesh D S. Modeling and optimization of parameters of flow rate of paddy rice grains through the horizontal rotating cylindrical drum of drum seeder. Computers and Electronics in Agriculture, 2009; 65(1): 26-35.

[9] Yi S J, Liu Y F, Wang C, Tao G X, Liu H Y, Wang R H. Experimental study on the performance of bowl-tray rice precision seeder. Int J Agric \& Biol Eng, 2014; 7(1): $17-24$.

[10] Zhang G Z, Zang Y, Luo X W, Wang Z M, Zhang Q, Zhang S S. Design and indoor simulated experiment of pneumatic rice seeder. Int J Agric \& Biol Eng, 2015; 8(4): 10-17.

[11] Zhai J B. Design and experiment of pneumatic precision hill-drop drilling seed metering device for rice budded seed. Wuhan: Huazhong Agricultural University, 2015. (in Chinese)

[12] Tian L Q, Wang J W, Tang H, Li S W, Zhou W Q, Shen H G. Design and performance experiment of helix grooved rice seeding device. Transactions of the CSAM, 2016; 47(5): 46-52. (in Chinese)

[13] Markauskas D, Kacianauskas R. Investigation of rice grain flow by multi-sphere particle model with rolling resistance. Granular Matter, 2011; 13(2): 143-148.

[14] Li J, Webb C, Pandiella S S, Campbell G M. Discrete particle motion on sieves-a numerical study using the EDEM simulation. Powder Technology, 2003; 133(1-3): 190-202.

[15] Fleissner F, Gaugele T, Eberhard P. Applications of the discrete element method in mechanical engineering. Multibody System Dynamics, 2007; 18(1): 81-94.

[16] Haiji Technology. Detailed explanation of EDEM software based on discrete element technology. CAD/CAM and Manufacturing Informatization, 2012; 5: 36-40. (in Chinese)

[17] Woo S M, Uyeh D D, Sagong M S, Ha Y S. Development of seeder for mixed planting of corn and soybeans. Int $\mathbf{J}$ 
Agric \& Biol Eng, 2017; 10(3): 95-101.

[18] Van Liedekerke P, Tijskens E, Dintwa E, Rioual F, Vangeyte J, Ramon H. EDEM simulations of the particle flow on a centrifugal fertilizer spreader. Powder Technology, 2009; 190(3): 348-360.

[19] Zhou H B. Research and application on key technologies of precision seeder for tray nursing seedlings of rice. Changchun: Jilin University, 2009. (in Chinese)

[20] Wang J W, Tang H, Wang J F, Li X, Huang H N. Optimization design and experiment on ripple surface type pickup finger of precision maize seed metering device. Int $\mathbf{J}$
Agric \& Biol Eng, 2017; 10(1): 61-71.

[21] Zhao Z, Wu Y F, Yin J J, Tang Z. Monitoring method of rice seeds mass in vibrating tray for vacuum-panel precision seeder. Computers and Electronics in Agriculture, 2015; 114: $25-31$.

[22] Zeng X Y. The research in mechanization planting ways and seeding ratios at different early direct seeding rice. Nanchang: Jiangxi Agricultural University, 2013. (in Chinese)

[23] GB/T6973-2005. Test method of single grain planter, 2005. (in Chinese) 